\title{
A PCR-based screening program to assess the prevalence of Taylorella equigenitalis in breeding stallions in South Africa
}

\author{
M.L. Schulman ${ }^{1}$, C.E. May ${ }^{1}$, C. Joone ${ }^{1}$, M. Monyai ${ }^{1}$, C. Gerstenberg ${ }^{2}$, R. Naidoo ${ }^{2}$, J. Pienaar ${ }^{2}$, \\ and A.J. Guthrie ${ }^{1}$ \\ ${ }^{1}$ Faculty of Veterinary Science, University of Pretoria, Onderstepoort, South Africa, \\ ${ }^{2}$ Directorate Animal Health, Department Agriculture, Forestry and Fisheries, Pretoria, Gauteng, South Africa
}

The first outbreak of Contagious Equine Metritis (CEM) due to Taylorella equigenitalis in South Africa was reported to the OIE in May 2011 subsequent to importation of a stallion, the index case. Two additional positive stallions were identified on an initial trace-back. The outbreak-response prompted determination of the national prevalence and distribution of CEM. A nation-wide PCR-based screening of all breeding stallions motivated by a previous outbreak report [1] was implemented via a mandatory CEM-negative clearance certificate prior to use for natural breeding or semen collection. Compliance from breeders was facilitated by developing a web-based system providing an easily-accessed, rapid and costeffective sampling, testing and reporting process on www.cemsa.co.za. A submission form, information, a breed-indexed list of stallions achieving CEM-clearance and a method for obtaining and submitting two sets of swabs (with an interval $>7 d$ ) from the external genitalia were accessible on the website. A duplex PCR was chosen as the assay method due to potential for submission of samples with minimal restrictions on transit time and temperature criteria and rapid, high throughput, cost-effi-ciency and reported sensitivity $[1,2]$. A clearance certificate was issued via the website after negative results from both sets of samples. Data recording stallion identity, breed, country of origin, location, sample dates and PCR results were analysed from samples received in the interval 15 August 2011-31 March 2012. 1718 Stallions representing 35 breeds from all nine provinces of South Africa were screened. The breed representation ranged from 1 to 493. The screening identified 33 suspected positive stallions subsequently confirmed positive on bacteriology. All were located in Gauteng except for one (Western Cape) and all linked to a single artificial breeding facility in Gauteng. Two, missed by the initial trace-back investigation, had been present at this facility before the index stallion, con-firming the presence of CEM in the country prior to this outbreak's detection. The innovative web-based screening provided a representative survey of the CEM status of the national population, unique demographic data and estab-lished the presence of CEM prior to importation of the index case. PCR, demonstrated as sensitive, enhanced sample submission criteria, turn-around times, reporting and cost-efficiency. 


\section{References}

[1] Erdman MM, Creekmore LH, Fox PE, Pelzel AM, Porter-Spalding BA, Aalsburg AM, Cox LK, Morningstar-Shaw BR, Crom RL. Diagnostic and epidemiologic analysis of the 2008-2010 investigation of a multi-year outbreak of contagious equine metritis in the United States. Prev Vet Med 2011;101:219-28.

[2] Wakeley PR, Errington J, Hannon S, Roest HIJ, Carson T, Hunt B, Sawyer J, Heath P. Development of a real time PCR for the detection of Taylorella equigenitalis directly from genital swabs and discrimina-tion from Taylorella asinigenitalis. Vet Micro 2006;118:247-54. 